\title{
Editorial
}

\section{Innovative Separation and Quality Control Strategies Applied to Mixed Polyolefins Waste}

The idea to write this Special Issue originated as I am involved in the running European FP7 Collaborative Project "Magnetic Sorting and Ultrasound Sensor Technologies for Production of High Purity Secondary Polyolefins from Waste (W2Plastics)" Grant Agreement No. 212782, specifically addressed to develop a number of novel concepts and an innovative operative approach finalized for recovering high-purity polyolefins from complex wastes at low cost.

Plastic waste recovery represents one of the key issues of recycling, not only from an environmental perspective, but also from a scientific-technical point of view. Plastic wastes, for their chemical physical attributes, represent, in fact, one of the most challenging materials to process in order to perform a correct separation of the different constituting polymers. The difficulties, influencing the separation-sorting strategies, being linked to several factors related both to polymers intrinsic characteristics (e.g. polymeric chain and structure) and utilized additives (e.g. fillers, pigments, etc.). Polyolefins represent a very important family of polymers, constituting more than a third of the total plastics consumption in Europe (EU). Complex wastes provide the vastest, presently unused potential resource of secondary polyolefins. In spite of that, polyolefins, such as polypropylene (PP), high density polyethylene (HDPE) and low density polyethylene (LDPE) are the least recycled plastic materials. Recycling technologies in this field need to be extremely powerful, since they must be relatively simple to be cost-effective, but also accurate enough to create high-purity products.

In this Special Issue are thus reported procedures and solutions adopted to reach the previous mentioned goal embedding not only the logic of understanding: why it works ('know-why') but also how a certain goal can be accomplished ('know-how'). A detailed knowledge of the market for streams of end-of-life products (supply side) and the market for recycled raw materials (demand side) are also always considered and evaluated to optimize the practical implementation of the logics inside the processing-recycling-chain.

The Special Issue starts with the paper by F. Di Maio, P. Rem, H. Bin, S. Serranti and G. Bonifazi "The W2Plastics Project: Exploring the Limits of Polymer Separation", reporting and analyzing the limits actually present in polymers separation as they result from Waste from Electric and Electronic Equipment (WEEE), Construction and Demolition Waste (CDW), household waste and Automotive Shredder Residue (ASR). Furthermore the definition of innovative evaluation criteria, processes and quality-inspection-control-strategies/logics (QICSL), in close conjunction with market requirements, was also outlined.

A correct separation is obviously always linked to knowledge of the characteristics of what have to be separated. In this perspective an in depth analysis is presented in the second paper by B. Vajna, K. Palásti, B. Bodzay, A. Toldy, S. Patachia, R. Buican, C. Catalin and M. Tierean "Complex Analysis of Car Shredder Light Fraction", based on FT-IR and Raman spectroscopy of different plastic feed waste streams, PP and PE recovered fractions, and related polluting materials. The composition and quality of the different separated fractions in respect of PP, PE and contaminants has been established.

In the third paper contributed by S. Serranti and G. Bonifazi "Post-consumer polyolefins (PP-PE) recognition by combined spectroscopic sensing techniques", the same spectroscopic techniques (FT-IR and Raman) have been then compared with a new analytical technique based on HyperSpectral Imaging (HSI). HSI can represent an optimal, reliable and low costs approach to perform and on-line control of the different "plastic streams" as they result from the different processing stages.

But what is the degree of the innovation required to maximize PP and PE recovery in a full economic perspective and inside a sector usually reluctant to accept new approaches? The answer is given in the fourth paper, by E.J. Bakker, A.J. Berkhout, L. Hartmann and P.C. Rem "Turning Magnetic Density Separation into Green Business using the Cyclic Innovation Model", utilizing new process logics and equipments adopting a non-linear approach. Based on the new conceptual framework outlined in the paper, the discussion of the problems related to the implementation of an innovative separation process, the Magnetic Density Separation (MDS) and related innovative control approaches, is introduced.

MDS separation technology has to face practical problems related to both materials physical-chemical characteristics and implemented separation architecture. Such problems are magnified if the materials to separate are very close in the property (density) utilized as discriminating parameter. The accuracy of sink-float separations in water-based media, such as MDS, can be in fact compromised by tiny air-bubbles attached to the surface of hydrophobic materials in the feed. In the fifth paper, by $B$. Hu, N. Fraunholz and P.C. Rem "Wetting Technologies for High-Accuracy Sink-Float Separations in Water-Based Media", a new wetting process to eliminate air bubbles was thus developed allowing a better utilization of MDS.

Together with particles wettability another important problem to face, when MDS has to be applied, is the control of the waterbased magnetic liquid. The sixth paper, authored by B. Hu, K. van Beek, A. Bosman, P.C. Rem, E.J. Bakker and F. Di Maio "Magnetization Control of Magnetic Liquids for Sink-Float Separations" deals with this subject. Different mixing tests have been thus performed and magnetization levels were compared with theoretical models. A measurement tool, based on a magneto-gravimetric principle, was also developed in order to detect and control fluctuations of the magnetic fluid in an industrial environment. 
With reference to MDS a key point of the method is the different plastics particles stratification according to their density and their further collection at different depths in an efficient way. To understand the behaviour of these materials a modelling approach was followed in the seventh paper by G. Houzeaux, C. Samaniego, H. Calmet, R. Aubry, M. Vazquez and P.C. Rem "Simulation of Magnetic Fluid applied to Plastic Sorting", in order to simulate particle paths into the water flow inside the separation device and to understand the separation mechanisms and to optimize its configuration.

What about the control of the different stages of the process and more specifically of the different plastic waste flow streams fed to the MDS and produced by the MDS? An innovative separation process, as the MDS, requires a correspondent set of equally new and sophisticated control actions able to perform a correct, reliable and low cost detection of flow streams composition. In this perspective two different monitoring actions, and related control logics, are proposed in the last two papers. The first one is developed in the paper by M.C.M. Bakker and S.A: Sanaee "Capabilities of Ultrasound for Monitoring and Quantitative Analysis of Polyolefin Waste Particles in Magnetic Density Separation (MDS)" and is based on ultrasound monitoring, the second one is introduced in the paper contributed by S. Serranti, A. Gargiulo and G. Bonifazi "The Utilization of Hyperspectral Imaging for Impurities Detection in Secondary Plastics" and is related to the application of HSI for plastic waste streams quality control.

The Special Issue provides an opportunity to share with the research community, working in the area of waste recycling, the knowledge and experience carried out until now inside the above mentioned European Project.

I would like to conclude this editorial thanking all the authors and reviewers for their contribution to this Special Issue.

Silvia Serranti

(Guest Editor)

Sapienza University of Rome Department of Chemical Engineering Materials \& Environment Via Eudossiana 18, 00184 Rome

Italy

Email: silvia.serranti@uniroma1.it

(C) Silvia Serranti; Licensee Bentham Open.

This is an open access article licensed under the terms of the Creative Commons Attribution Non-Commercial License (http://creativecommons.org/licenses/by$\mathrm{nc} / 3.0 /$ ) which permits unrestricted, non-commercial use, distribution and reproduction in any medium, provided the work is properly cited. 